Pesq. Vet. Bras. 35(12):989-996, dezembro 2015 DOI: $10.1590 / \mathrm{S} 0100-736 \mathrm{X} 2015001200008$

\title{
Avaliação da epífora de cães usando dacriocistografia e tomografia computadorizada ${ }^{1}$
}

\author{
Nivea M.G. Vieira ${ }^{2 *}$, Joaquim J.T. Ranzani ${ }^{3}$, Cláudia V.S. Brandão ${ }^{3}$, Daniela N. Cremonini ${ }^{3}$, \\ Silvana A. Schellini ${ }^{3}$, Carlos R. Padovani ${ }^{3}$, Luis C. Vulcano ${ }^{3}$ e Mariana F. Almeida ${ }^{3}$
}

\begin{abstract}
Vieira N.M.G., Ranzani J.J.T., Brandão C.V.S., Cremonini D.N., Schellini S.A., Padovani C.R., Vulcano L.C. \& Almeida M.F. 2015. [Epiphora assessment of dogs with dacryocystography and computed tomography.] Avaliação da epífora de cães usando dacriocistografia e tomografia computadorizada. Pesquisa Veterinária Brasileira 35(12): 989-996. Serviço de Oftalmologia Veterinária, Departamento de Cirurgia e Anestesiologia, Faculdade de Medicina Veterinária e Zootecnia, Universidade Estadual Paulista, Distrito de Rubião Jr s/n, Botucatu, SP 18618-970, Brazil. E-mail: niveamgv@yahoo.com.br

Epiphora evaluation can be done through clinical exams, lachrymal excretion tests, contrasted exams, like radiologic evaluation and tomography studies; those last two allow a detailed analysis of the structures. The present study had as the objective to evaluate the lacrimal drainage system through radiography and contrasted computed tomography, which were made with animals presenting epiphora, comparing them to healthy animals, without the mentioned condition. Twenty dogs were used for tests, from a variety of breeds and coatings, males and females, weight varying from $1 \mathrm{~kg}$ to $20 \mathrm{~kg}$, ages varying from 0.7 to 8 years old, presenting epiphora (epiphora group - GE*). As a control group (GC*) 15 dogs were used, with no clinical alterations of naso-lachrymal drainage system, from different breeds and coatings, males and females, weighing from $1 \mathrm{~kg}$ to $20 \mathrm{~kg}$ and ages varying from 0.7 to 8 years old. It was propoused the division of the lacrimal drainage system in four regions. On region 1 , the GE had $29(76.3 \%)$ animals with dilation visualized by the RX and $32(84.2 \%)$ by CT, the GC, $4(12.5 \%)$ in the RX and 1 (3.1\% ) CT showed dilation. In region 3, 13 (34.2\%) dogs showed dilatation of the DLN in RX and $14(36.8 \%)$ on CT, and 21 (55.3\%) nasolacrimal duct communication with the sinus and the X-ray $28(73.7 \%)$ by CT. For the CG, 15 (46.9\%) the X-ray and $22(68.7 \%)$ by CT had nasolacrimal duct communication with the nasal sinus. It has been concluded that dilations can also be observed in some dogs with no clinical signs of disease; nasolacrimal duct communication to sinus does not indicate epiphora causing alteration, as it is present in animals with and without affection; the present study data confirm that DCG itself can provide important informations, in a similar manner to CT, and should resort to it just in case of doubts about bone lesions, fractures and foreign bodies undetected at first.
\end{abstract}

INDEX TERMS: Radiography, computed tomography, dacryocystography, epiphora, dogs.

RESUMO.- A avaliação da epífora pode ser feita por exames clínicos, testes de excreção lacrimal, exames contrastados, como avaliação radiológica e estudo tomográfico; estes

\footnotetext{
${ }^{1}$ Recebido em 17 de setembro de 2015.

Aceito para publicação em 24 de novembro de 2015.

${ }^{2}$ Pós-Graduada, Serviço de Oftalmologia Veterinária, Departamento de Cirurgia e Anestesiologia, Faculdade de Medicina Veterinária e Zootecnia (FMVZ), Rua Gonçalves Dias 845, Bloco I, Apto 34, Vila Gabriel, Sorocaba, SP 18081-040, Brasil. *Autor para correspondência: niveamgv@yahoo. com.br

${ }^{3}$ Serviço de Oftalmologia Veterinária, Departamento de Cirurgia e Anestesiologia, FMVZ, Universidade Estadual Paulista (Unesp), Distrito de Rubião Jr s/n., Botucatu, SP 18618-970, Brasil. E-mail: quim@fmvz.unesp.br
}

dois últimos permitem análise minuciosa das estruturas. 0 presente estudo teve como objetivo avaliar a via lacrimal excretora com uso de radiografia e tomografia computadorizada contrastadas, feitas em animais com epífora, comparando-se com animais sadios, sem essa afecção. Foram utilizados 20 cães, de raças e pelagens variadas, machos e fêmeas, com peso de 1 a $20 \mathrm{~kg}$, com 0,7 a 8 anos de idade, apresentando epífora (grupo epífora - GE). Como grupo de controle (GC), foram utilizados 15 cães, sem alterações clínicas de drenagem do sistema lacrimonasal de raças e pelagens variadas, machos e fêmeas, pesando 1 a $20 \mathrm{~kg}$, com 0,7 a 8 anos. Foi proposta a divisão do sistema de drena- 
gem lacrimal em quatro regiões. Na região 1, o GE teve 29 (76,3\%) animais com dilatação visibilizada pelo R-X e 32 $(84,2 \%)$ pela TC; no GC, $4(12,5 \%)$ no R-X e $1(3,1 \%)$ na TC demonstraram dilatação. Na região 3, 13 (34,2\%) cães evidenciaram dilatação do DLN no R-X e 14 (36,8\%) na TC; e 21 (55,3\%) comunicação do ducto lacrimonasal com o seio nasal pelo R-X e 28 (73,7\%) pela TC. Já no GC, 15 (46,9\%) pelo R-X e $22(68,7 \%)$ pela TC possuíam comunicação do ducto lacrimonasal com o seio nasal. Concluiu-se que: dilatações também podem ser observadas em alguns cães sem sinal clínico da afecção; comunicação do ducto lacrimonasal com o seio nasal não indica alteração causadora de epífora, pois está presente em animais com e sem afecção; os dados do presente estudo confirmam que o exame DCG por si pode dar importantes informações, de forma semelhante à TC, devendo-se recorrer à ela apenas quando houver dúvidas sobre lesões ósseas, fraturas e corpos estranhos não detectados pelo primeiro.

TERMOS DE INDEXAÇÃO: Radiografia, tomografia computadorizada, dacriocistografia, epífora, cães.

\section{INTRODUÇÃO}

O sistema de drenagem lacrimal do cão compreende os pontos lacrimais, canalículos lacrimais, saco lacrimal, ducto lacrimonasal e óstio nasal, estruturas para as quais converge a lágrima que, então, segue para a narina (Roberts et al. 1974, Diesem 1981, Slatter 1990b, Severin 1991, Habin 1993). (Fig.1)

Epífora é a mancha ocular crônica, de cor ferruginosa comum em determinadas raças (Mills \& Meyer 2006, Zaldivar et al. 2006) e desenvolve-se devido à obstrução do fluxo lacrimal no sistema lacrimonasal no qual o volume de lágrimas excede o sistema de drenagem normal e deve ser diferenciada da superprodução de lágrimas, denominado lacrimejamento ativo (Gelatt 2003).

A dacriocistografia é o exame contrastado pelo qual se avalia a via lacrimal excretora, observando-se o local exato

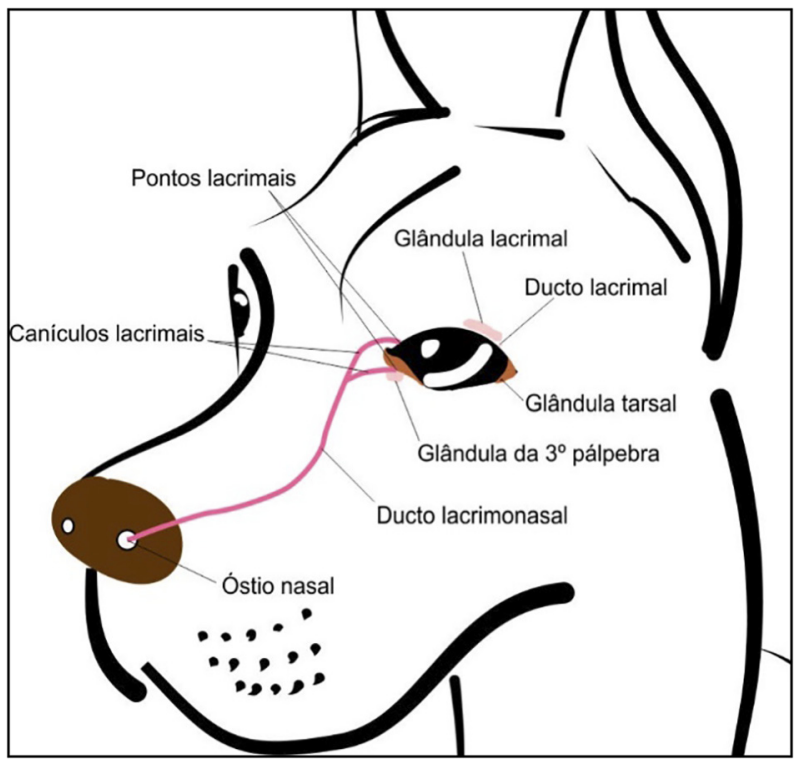

Fig.1. Esquema do sistema de drenagem lacrimonasal no cão. da obstrução, além de estenoses, fístulas, divertículos, defeitos de preenchimento causados por cálculos ou tumores (Araf 2004, Kanski 2004a, 2004b). Esse exame não é utilizado apenas para avaliar a patência do ducto lacrimonasal, mas também para revelar a localização anatômica do problema (Gelatt et al. 1972). Ou seja, a dacriocistografia (DCG) pode determinar com precisão a localização da obstrução ou estenose da via lacrimal excretora (Lloyd \& Welham 1974). Este é, portanto, exame muito útil para a avaliação anatômica do sistema quando usado contraste oleoso (Schellini et al. 2005).

A técnica em cães é realizada com o paciente sob anestesia geral e em decúbito lateral. Pode ser realizada, também, exposição radiográfica dorsoventral (Slatter 1990a). Além desse método, existe a possibilidade de realizarem-se radiografias nasais com a boca aberta, lateral e ventro-dorsal (Gelatt 2003).

Inicialmente, os pontos lacrimais inferiores são dilatados com dilatador de ponto. Cateteres são inseridos nos canalículos inferiores em ambos os lados (os pontos lacrimais superiores podem ser usados alternativamente). Geralmente, $1 \mathrm{ml}$ de contraste iodado viscoso é simultaneamente injetado em ambos os ductos em sentido caudo-rostral, e as radiografias são feitas lateral ou ventrodorsalmente. Cinco minutos após a injeção, é realizada nova radiografia para avaliar o efeito da gravidade sobre a drenagem lacrimal (Gelatt 2003). Quando utilizado o contraste oleoso, radiografias podem ser tiradas após 30-40min (dacriocistografia de retardo) para avaliar a presença de contraste residual, sugerindo alteração funcional da drenagem lacrimal (Araf 2004, Kanski 2004a, 2004b). As radiografias são obtidas conforme o contraste passa pelo ducto lacrimonasal; perfurações ou bloqueios deste podem ser detectados nestas imagens (Gelatt 2003).

Na medicina a ausência do contraste na cavidade nasal indica obstrução anatômica, cujo sítio é geralmente evidenciado. Uma dacriocistografia (DCG) normal na presença de epífora indica obstrução parcial ou falência da bomba lacrimal (Kanski 2004a, b). Na veterinária, o contraste deve ser observado também na cavidade oral (Gelatt 2003).

Dacriocistografia por tomografia computadorizada oferece excelente resolução das imagens do canal lacrimonasal, por fornecer importantes informações para o diagnóstico e tratamento de doenças lacrimais (Nykamp et al. 2004, $\mathrm{Yu}$ et al 2013). Imagem de tomografia computadorizada é primariamente usada em avaliações de patologias oculares, secundariamente usada pra avaliar viabilidade, imagem detalhada, e tempo curto de escaneamento. Recentemente, numerosos estudos têm usado TC como uma ferramenta primária no detalhamento da anatomia do sistema de drenagem nasolacrimal e, como as variações, podem evidenciar as disfunções de drenagem (Groell et al 1997, Lee et al 2011, Lefebvre \& Freitag 2012, Ramey et al 2013, Czyz et al 2015). A maioria dessas pesquisas tem focado nas variações de estruturas, como no diâmetro e área do ducto nasolacrimal (DNL), volume nasolacrimal, ou ângulo do ducto em relação ao assoalho nasal (Lefebvre \& Freitag 2012).

A TC utiliza ondas de raios-X para a obtenção de diferentes densidades teciduais de secções, formadas por um 
computador. Os cortes podem ser axiais e coronais (frontal), mas não sagitais. Lesões com vascularização intrínseca (massas) podem ser melhor visualizadas com o uso de contrastes iodados (Araf 2004, Kanski 2004a, 2004b).

A dacriocistografia por tomografia computadorizada tem mostrado ser a técnica mais confiável para descrever a anatomia do ducto lacrimonasal com alta resolução espacial (Rached et al. 2007).

Tendo em vista a grande procura dos proprietários por oftalmologistas veterinários, devido aos casos de extravasamento da lágrima decorrente de prováveis alterações no trajeto anatômico da via lacrimal em cães, o presente estudo teve como objetivo avaliar a via lacrimal excretora. Após várias tentativas, usou-se técnicas específicas desenvolvidas durante esse trabalho, especialmente para obtenção de imagens limpas, sem interferência do excesso do contraste pelo extravasamento dentro de seios nasais nos exames de radiografia e tomografia computadorizada contrastadas, em cães com epífora crônica. Foram canulados os pontos lacrimais com cateter e feitas injeções de contraste dentro de todo o trajeto do sistema de drenagem lacrimal, concomitante ao momento de obtenção das imagens. Na radiografia, o contraste foi injetado manualmente, muito lentamente, por uma pessoa no transcorrer do exame. Na tomografia computadorizada, devido a radiação e a impossibilidade da presença de uma pessoa durante os exames, usou-se uma bomba de infusão peristáltica injetando o contraste, também de forma bem lenta, no transcorrer do exame. Novamente, após várias tentativas, desenvolveu-se uma forma de obtenção de imagens sem interferência do contraste: colocou-se esparadrapo logo abaixo das pálpebras inferiores e no focinho, contornando inferiormente os pontos lacrimais inferiores, recoberto por papel contact para evitar que o contraste aderisse aos pelos e para facilitar a limpeza do mesmo. Em seguida, comparou-se as imagens dos animais com e sem epífora.

\section{MATERIAL E MÉTODOS}

Foram constituídos dois grupos experimentais: o grupo com epífora (GE) e o grupo de controle (GC). No GE, foram avaliados 20 cães, de raças e pelagens variadas, machos e fêmeas, com pesos entre 1 e $20 \mathrm{~kg}$, de 0 a 8 anos de idade, apresentando epífora crônica. Como GC, foram utilizados 15 cães, sem alterações clínicas do sistema lacrimonasal, de raças e pelagens variadas, machos e fêmeas, pesando 1 e $20 \mathrm{~kg}$, com 0 a 8 anos.

O GE foi composto por busca ativa em clínicas veterinárias e pet shops e por cães cujos proprietários procuraram o atendimento oftalmológico do serviço de Oftalmologia Veterinária do Hospital Veterinário da Faculdade de Medicina Veterinária e Zootecnia (FMVZ), Universidade Estadual Paulista, Campus Botucatu, entre julho de 2007 e julho de 2009.

0 critério de inclusão para esses animais foi a presença de histórico de mancha amarronzada na face, a partir do canto nasal do olho, associada a aspecto estético indesejável, confirmado por exame clínico oftalmológico.

Foram excluídos animais com epífora crônica que apresentavam lacrimejamento ativo (distiquíase, úlceras corneanas, triquíase, entrópio) ou epífora por obstrução mecânica do sistema lacrimal (protrusão de glândula da terceira pálpebra, entrópio de canto nasal inferior, dacriocistites).

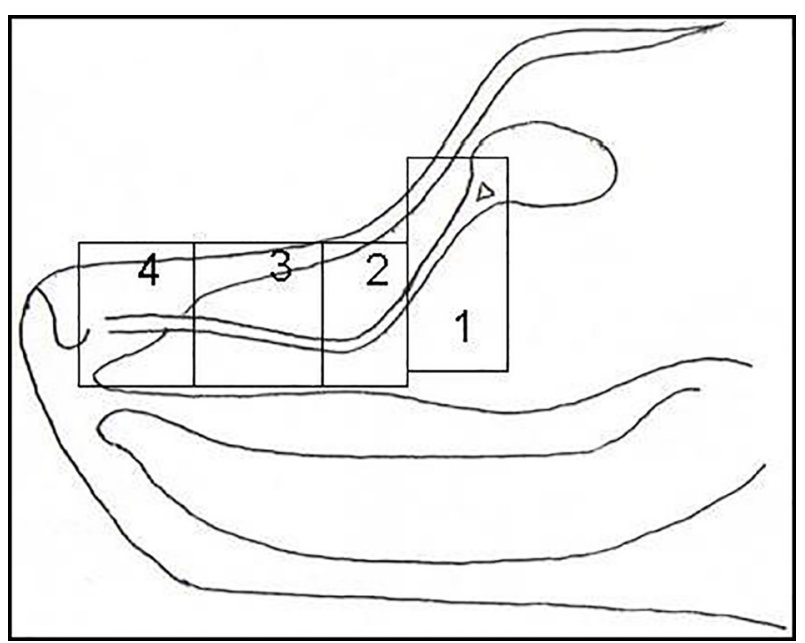

Fig.2. Figura esquemática com as regiões divididas em 1, 2, 3 e 4. A Região 1 compreende os canalículos e o saco lacrimonasal; Região 2, maior tortuosidade do ducto de drenagem lacrimal; Região 3, caudal ao canino e onde situam-se os seios nasais acima do ducto; Região 4, cranial ao canino e final do DLN que desemboca no óstio nasal.

O GC foi constituído por cães que não possuíam sinais clínicos de extravasamento da lágrima, atendidos no Hospital Veterinário.

Aspectos éticos. Todos os critérios utilizados seguiram as normas para experimentação animal da Association for Research in Vision and Ophthalmology (ARVO) e foram aprovados pela Câmara de Ética em Experimentação Animal (Número do processo 129/2007) da Faculdade de Medicina Veterinária e Zootecnia, Unesp - Campus Botucatu.

Análise de resultados. Foi estudado o sistema de drenagem lacrimonasal, visibilizando o diâmetro dos canalículos, do saco lacrimal e do ducto lacrimonasal entre os animais que apresentam epífora e o grupo de controle. Foi analisado o percurso do contraste nesses animais, em relação às áreas de dilatação, estenose, desvio, agenesia ou obstrução de todo o sistema de drenagem lacrimonasal. Para ajudar o viés da subjetividade e imparcialidade das interpretações das imagens, obteve-se ajuda de dois revisores.

Na Figura 2, evidencia-se a divisão do sistema lacrimal excretor, proposta neste trabalho, para facilitar o estudo das alterações frente a interpretação radiográfica e tomográfica, classificando-o em quatro regiões.

As variáveis dilatação (D), comunicação do ducto lacrimonasal com a cavidade nasal (C), estenose (E), obstrução (O), tortuosidade (T) e massa e/ou abscesso (M) foram avaliadas nas diferentes regiões nos grupos GE e GC segundo número e porcentagem das alterações acima, considerando a ausência ou presença de epífora.

Técnica de dacriocistografia por radiografia contrastada e tomografia computadorizada. Todos os animais de ambos os grupos foram submetidos ao exame dacriocistográfico por radiografia e tomografia computadorizada. 0 protocolo anestésico adotado foi acepromazina ${ }^{4}$ na dose de $0,05 \mathrm{mg} / \mathrm{kg}$ IM e morfina 0,5 $\mathrm{mg} / \mathrm{kg}$ IM, como pré-anestésico seguido da anestesia dissociativa de tiletamina e zolazepan ${ }^{5}$ na dose de $5 \mathrm{mg} / \mathrm{kg}$ IV, com repiques de $1-2 \mathrm{mg} / \mathrm{kg}$, quando necessários.

Após a anestesia, os animais foram posicionados na mesa do aparelho de Raio- $X^{6}$ na posição látero-lateral (Fig.3) de ambos os lados. Foi instilado o colírio anestésico ${ }^{7}$ e dilatou-se o ponto lacri-

\footnotetext{
${ }^{4}$ Acepran ${ }^{\circledR} 0,2 \%$ - Univet, Brasil.

${ }^{5}$ Zoletil $^{\circledR}$ - Virbac, EUA.

${ }^{6}$ Máquina Shimadzu modelo EZY-RAD , Japão.

${ }^{7}$ Anestalcon ${ }^{\circledR}$ - Alcon , Brasil.
} 


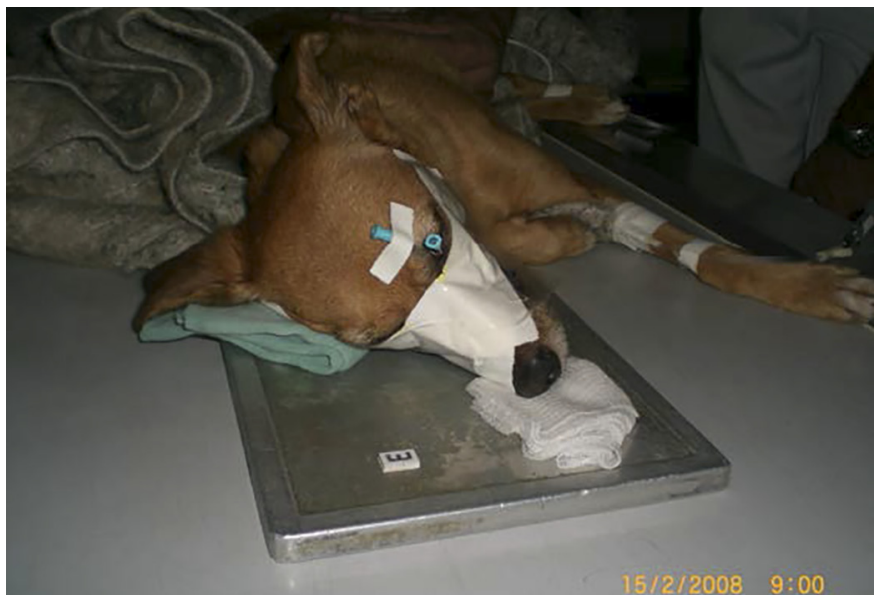

Fig.3. Imagem fotográfica de animal posicionado na mesa de raios-X em decúbito látero-lateral.

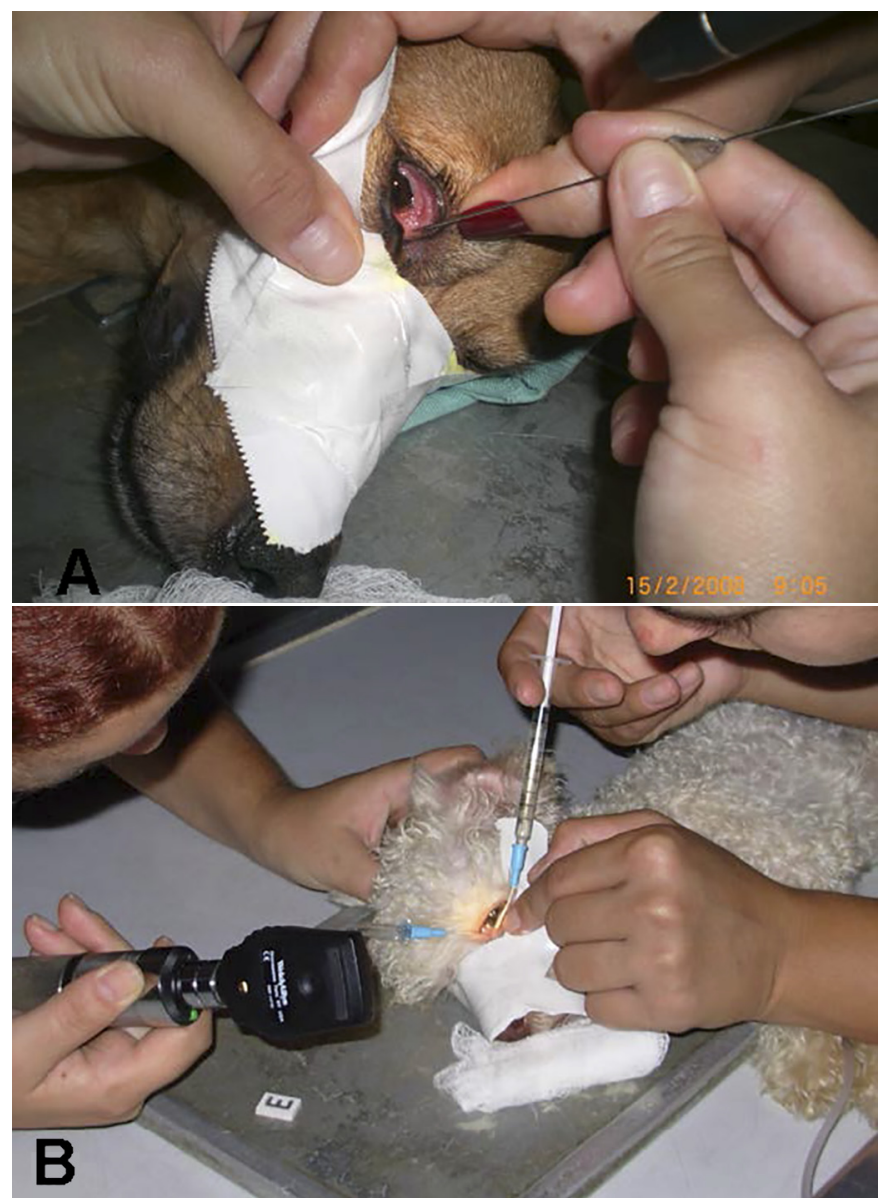

Fig.4. Dilatação do ponto lacrimal. (A) Dilatação do ponto lacrimal com o dilatador de ponto lacrimal. (B) Fluxo contínuo manual do contraste.

mal superior e inferior com o dilatador de ponto lacrimal. Em seguida, o exame radiográfico de todo o aparelho lacrimal (dacriocistografia) foi realizado pelo fluxo contínuo manual do contraste iodado à base de óleo de papoula ${ }^{8}$, pelo ponto lacrimal inferior, antes e durante a radiografia; canulado com cateteres número 20 , 22, 24 (dependendo do diâmetro do ponto lacrimal de cada indivíduo) acoplada a uma seringa de $1 \mathrm{ml}$ (Fig.4).

\footnotetext{
${ }^{8}$ Lipiodol ${ }^{\circledR}$ U.F. - Guerbet, Brasil.
}

No exame radiográfico, foram utilizados os seguintes parâmetros: 1,8mA; 2,0mA e 2,2 mA e $43 \mathrm{kV} ; 45 \mathrm{kV}$ e $46 \mathrm{kV}$; tais parâmetros variaram entre os pacientes, dependendo de seu porte. 0 sistema lacrimonasal de cães foi delimitado radiograficamente pela injeção do contraste através do ponto lacrimal (Fig.5).

Em seguida, os pacientes foram posicionados na mesa do aparelho de tomografia computadorizada na posição dorso-ventral, com pálato perpendicular à mesa de scanner, para obtenção de imagem transversa da cavidade nasal (Fig.6), de ambos os lados. Foi instilado o colírio anestésico e dilatou-se o ponto lacrimal superior e inferior com o dilatador de ponto lacrimal. Pelo fato de o meio de contraste escoar do sistema muito rapidamente pela ação da gravidade, o que não ocorre em humanos, foi necessária
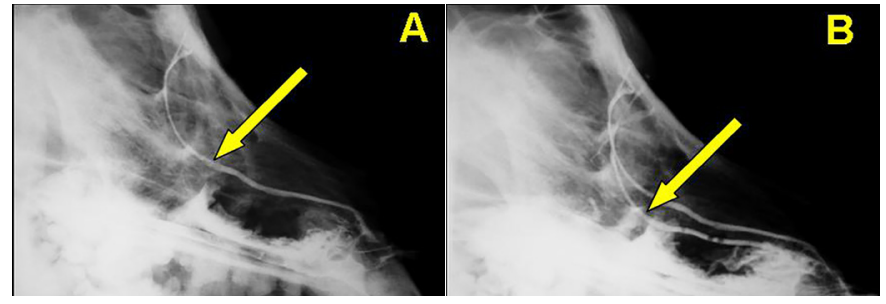

Fig.5. Imagens radiográficas da visibilização dos ductos lacrimonasais através de radiografia contrastada. (A) Ducto lacrimonasal direito (seta). (B) Ducto lacrimonasal esquerdo (seta) e resquício do contraste no ducto direito.
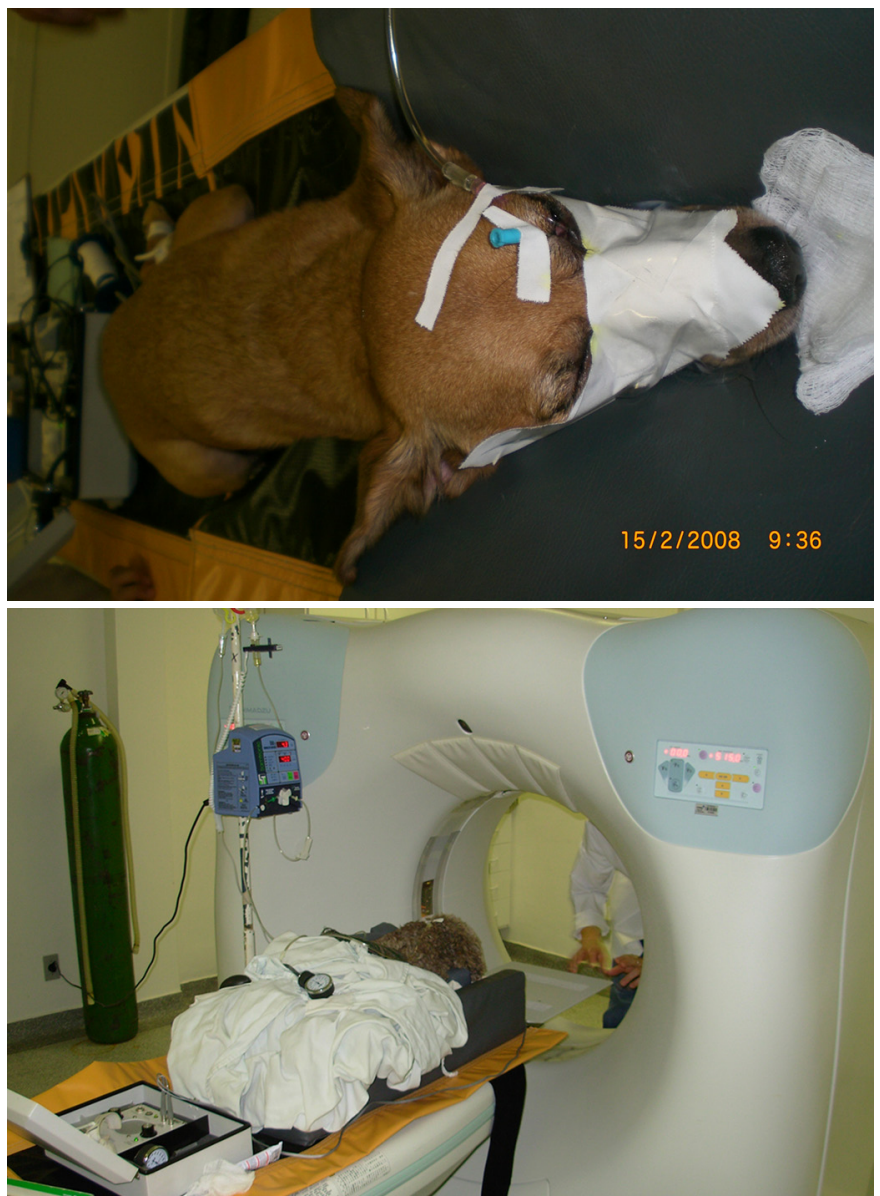

Fig.6. Imagens fotográficas de animal na mesa de tomografia computadorizada. (A) Posicionamento da cabeça do cão antes de entrar no tomógrafo. (B) Animal entrando no aparelho de tomografia computadorizada. 


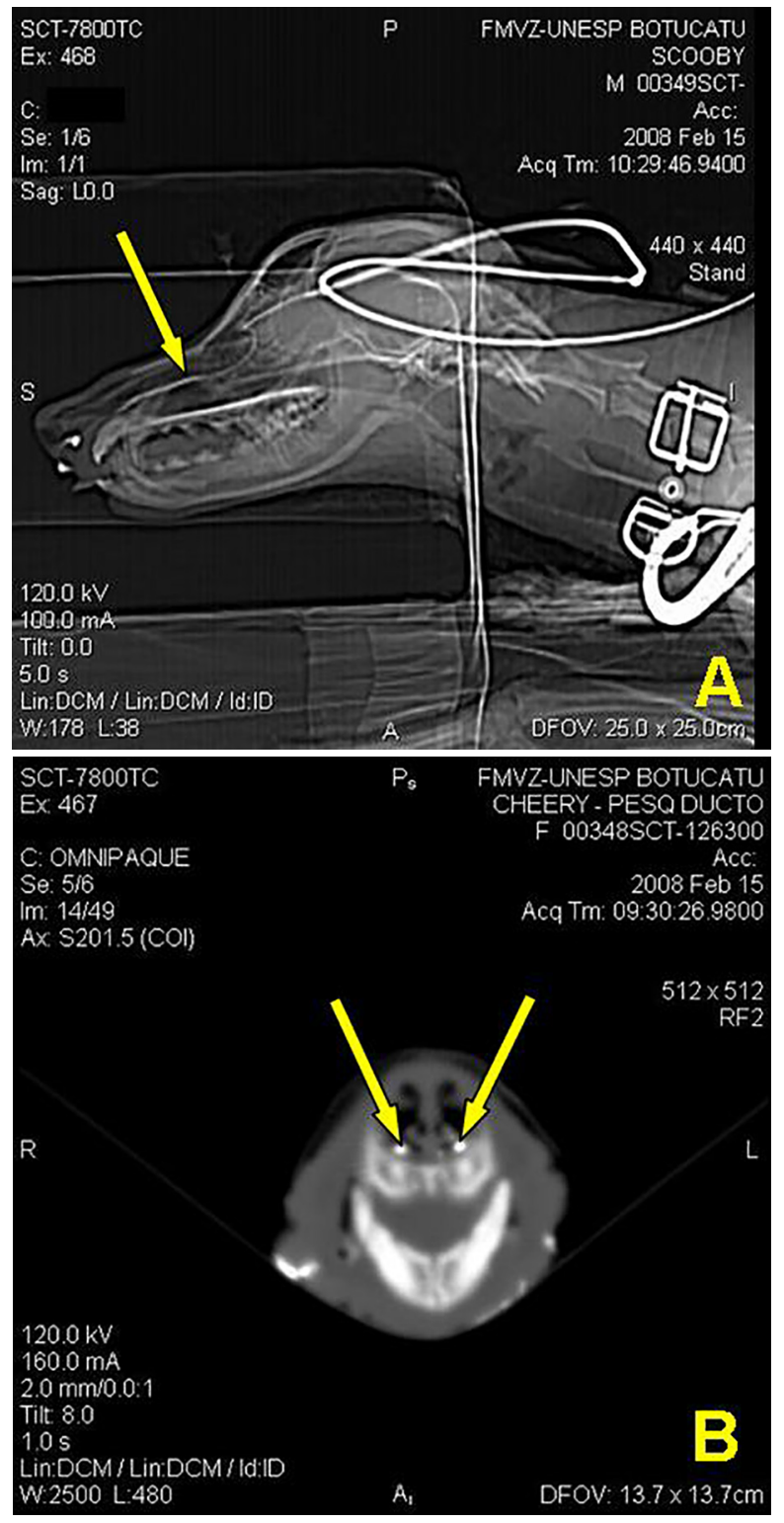

Fig.7. Imagens da tomografia computadorizada. (A) Scalt. (B) Passagem do contraste em ambos os ductos lacrimonasais em corte tomográfico.

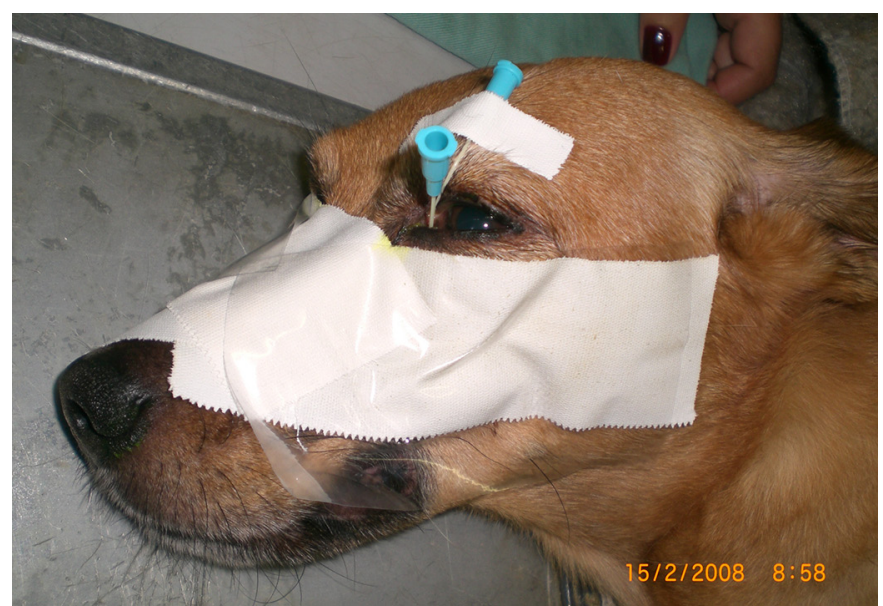

Fig.8. Imagem fotográfica da impermeabilização da região abaixo do ponto lacrimal (seta). injeção contínua do contraste com auxílio de bomba de infusão peristáltica, para adequada reconstituição das imagens.

No exame de tomografia computadorizada, foram utilizados os seguintes parâmetros: $160 \mathrm{~mA}, 120 \mathrm{kV}, 512$ o tamanho da matriz, com cortes variando entre $1 \times 1$ e $2 \times 2 \mathrm{~mm}$. Os cortes variaram entre os pacientes. As imagens foram disparadas na janela óssea (W 3.800, L 600) e uma janela do tecido mole (W 250, L 70). 0 ponto lacrimal foi canulado e 0,4 a $1,2 \mathrm{ml}$ do contraste foi injetado. 0 exame foi realizado durante a injeção do contraste. 0 tomógrafo ${ }^{9}$ utilizado é do tipo helicoidal e as imagens foram transferidas no formato DICOM para um work station a fim de posterior reconstrução das imagens gravadas em um CD (Fig.7).

Foi colocado esparadrapo logo abaixo das pálpebras inferiores e no focinho, contornando inferiormente os pontos lacrimais inferiores, recoberto por papel contact para evitar que o contraste aderisse nos pelos e para facilitar a limpeza do mesmo, evitando o obscurecimento da imagem radiográfica e tomográfica (Figura 8).

Após cada procedimento, o sistema lacrimonasal de cada cão foi lavado com soro fisiológico em jato para retirar o resíduo de contraste.

\section{RESULTADOS}

De acordo com os resultados obtidos quanto à avaliação do sistema lacrimonasal, pelo preenchimento do mesmo com contraste e exames de diagnóstico por imagem, feitos com uso de radiografias (R-X) e tomografias computadorizadas (TC), pode-se observar que:

\section{Região 1: canalículo e saco lacrimonasal}

No GE 7 (18,4\%) animais apresentaram dilatação do DLN visibilizado tanto no R-X como na TC.

De todos os animais estudados do GC, apenas $1(3,1 \%)$ deles apresentou estenose no DLN evidenciado apenas no R-X na região 1.

Região 2: maior tortuosidade sistema de drenagem lacrimal

O GE possuía $8(21,0 \%)$ animais com dilatação demonstradas pelo R-X e pela TC; 2 (5,3\%) estenose visibilizada apenas no R-X e $1(2,6 \%)$ tortuosidade percebida na TC.

No GC, novamente apenas $1(3,1 \%)$ cão apresentou estenose no DLN também evidenciado apenas no R-X.

Região 3: caudal ao canino e localização dos seios nasais acima do ducto

As imagens estudadas no GE demonstraram que no R-X 14 $(34,2 \%)$ e na TC $14(36,8 \%)$ cães evidenciaram dilatação do DLN; $21(55,3 \%)$ pelo R-X e $28(73,7 \%)$ pela TC comunicação do ducto lacrimonasal com o seio nasal (Fig.9); 2 (5,3\%) estenose visibilizado no R-X; 1 (2,6\%) obstrução pela TC; 1 (2,6\%) na TC observou-se massa e/ou abscesso; 1 (2,6\%) trajeto tortuoso pelo R-X.

Já no GC, 15 (46,9\%) pelo R-X e $22(68,7 \%)$ pela TC possuíam comunicação do ducto lacrimonasal com o seio nasal e $1(3,1 \%)$ pelo R-X estenose.

\section{Região 4: cranial ao canino e final do DLN onde desemboca} no óstio nasal

Finalmente, no GE, 29 (76,3\%) dos animais tinham dilatação visibilizada pelo R-X e 32 (84,2\%) pela TC; 3 (7,9\%) evidenciaram estenose apenas pelo R-X; obstrução $5(13,1 \%)$ pelo R-X e 1 $(2,6 \%)$ pela TC; $1(2,6 \%)$ trajeto tortuoso pelo R-X; dilatação mais estenose 1 (2,6\%) pelo R-X; dilatação mais tortuosidade $1(2,6 \%)$ pelo R-X; dilatação mais obstrução $1(2,6 \%)$ pelo R-X.

No GC, $4(12,5 \%)$ no R-X e $1(3,1 \%)$ na TC demonstraram dilatação (Fig. 10); 1 (3,1\%) estenose na TC.

\footnotetext{
${ }^{9}$ Máquina Shimadzu modelo SCT-7800TC, Japão.
} 


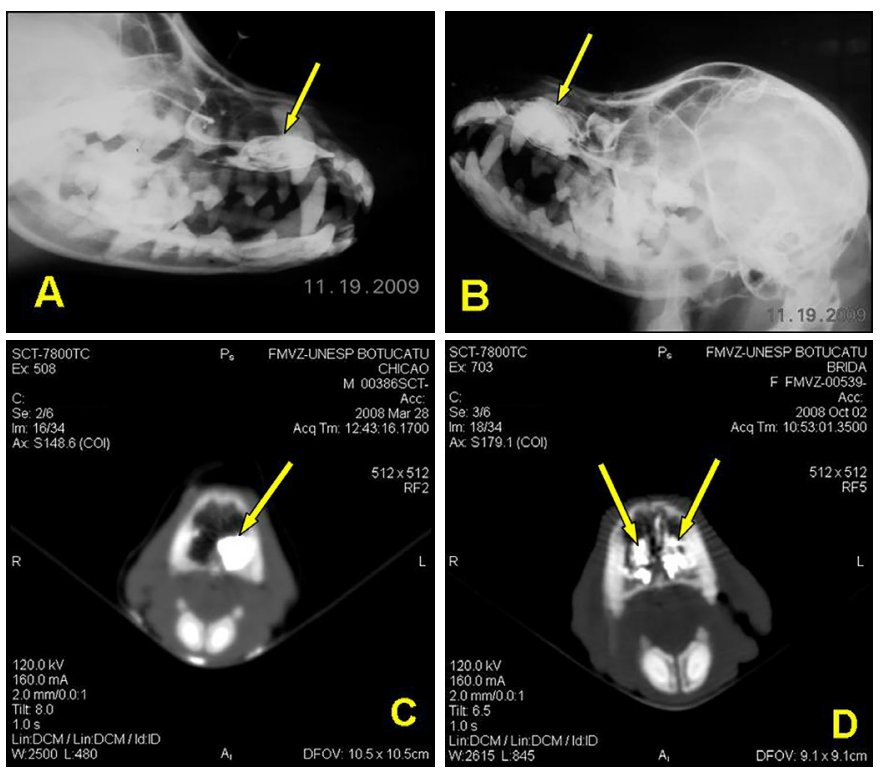

Fig.9. Imagens radiográficas e tomográficas da comunicação do ducto lacrimonasal com o seio nasal (setas). (A,B) Radiografias do contraste nos seios nasais; (C,D) Tomografias do contraste nos seios nasais.
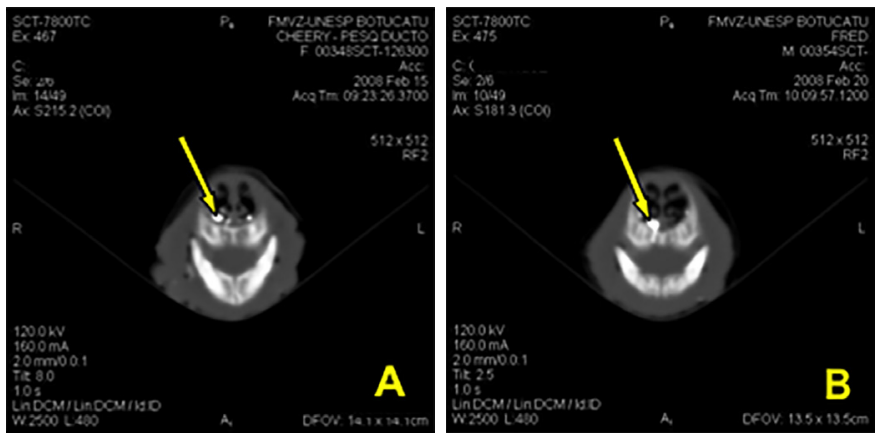

Fig.10. Imagens tomográficas de dilatação do ducto lacrimonasal em Região 4. (A,B) Corte tomográfico de dilatação do ducto lacrimonasal direito em cães distintos (setas).

\section{DISCUSSÃO}

Apesar de os exames de imagem serem bastante utilizados em humanos para o diagnóstico da epífora, e de ter-se possibilidade de utilizá-los há várias décadas, não se encontra, na literatura, com frequência, sua utilização em cães. Esses são exames muito importantes, com chances de demonstrar alterações anatômicas e estruturais da via lacrimal.

Visto que não houve na bibliografia consultada nenhuma divisão do DLN semelhante ao realizado em nosso trabalho, não foi possível fazer comparações e discussões com outros trabalhos.

O exame radiográfico contrastado realizado prestou-se como método de reconhecimento da anatomia e da localização das estruturas do sistema de drenagem lacrimal dos cães, proporcionando, também, a verificação da patência dessas estruturas, conforme também relatado por vários autores na Medicina e na Veterinária, como Caye et al. (1986), Slatter (1990a), Habin (1993), Barnett et al. (1995), Takano \& Mendonça Júnior (1996), Carneiro Filho (1997), Pereira et al. (1997), Shankar et al. (1999), Stades et al. (1999),
Wearne et al. (1999), Grahn (2002), Gelatt (2003), Winston \& Prasse (2003), Kleiner et al. (2004), Nykamp et al. (2004) e Silva (2004), que apontam a dacriocistografia como importante exame auxiliar. No presente trabalho, foi possível avaliar anatomicamente o sistema lacrimonasal também pela dacriocistografia por tomografia computadorizada contrastada, o que auxiliou na avaliação do sistema lacrimonasal em pacientes com alterações nas vias lacrimais concordando com os autores Costa et al. (1997) que afirmaram que a semiologia da via lacrimal excretora envolve dados da anamnese, avaliação anatômica e funcional. Esta avaliação é realizada pela dacriocistografia (DCG) contrastada, tomografia computadorizada ou ressonância magnética. Os resultados do presente trabalho também corroboram com os autores Rached et al. (2011), que concluíram que a DCG-TC é uma ferramenta de diagnóstico útil para a avaliação do sistema nasolacrimal. Nykamp et al. (2004) também defenderam que a TC é usada em epífora crônica e permite informações pertinentes que auxiliam no diagnóstico e/ou permitindo melhor apuramento no prognóstico.

Para esclarecer se existem alterações na patência do ducto lacrimonasal, após os exames funcionais do sistema lacrimonasal, os métodos complementares de diagnóstico por imagem, como a dacriocistografia por radiografia e tomografia computadorizada contrastadas são de grande utilidade na medicina veterinária. Porém, os dados do presente estudo confirmam que o exame DCG por si pode dar importantes informações, de forma semelhante à TC, devendo-se recorrer à ela apenas quando houver dúvidas sobre lesões ósseas, fraturas e corpos estranhos não detectados pelo primeiro. Um fator limitante desse último exame refere-se a seu alto custo quando comparado com o de radiografia. Boruchoff \& Boruchoff (1992) indicaram a tomografia computadorizada principalmente em pacientes com obstrução secundária a tumores ou má formação congênita dos tecidos circundantes.

Limitações desse estudo, também encontradas por Czyz et al 2015, incluem a interpretação subjetiva das imagens radiográficas e tomográficas e tendências na interpretação das imagens. Para minimizar esse viés na interpretação, assim como Czyz et al 2015, as imagens foram revisadas por outros avaliadores imparciais.

Nos animais utilizados no presente estudo, foi identificada, pelo exame radiográfico, uma segunda abertura do ducto lacrimonasal, além da abertura normal anatômica da narina (óstio nasal), o que foi também relatado por Stades et al. (1999), Gelatt (2003), Kleiner (2003) e Kleiner et al. (2004). Segundo Michel (1955), a função adequada do sistema lacrimonasal não depende apenas do diâmetro, mas também do seu curso. Michel (1955), Böhme (1992) e Gelatt (2003) relatam que, em cerca de $40 \%$ dos cães, há comunicação do ducto lacrimonasal com a cavidade nasal abaixo da concha nasal ventral na direção do dente canino.

Foi observado neste estudo que, após a irrigação do sistema lacrimonasal, os animais retornavam ao ambulatório após uma semana e continuavam com epífora. Segundo Long (1975) e Covitz et al. (1977), tratamento e prognóstico de doenças do sistema de drenagem lacrimal (epífora) não são muito satisfatórios. 
No presente estudo, utilizou-se o meio de contraste iodado a base de óleo papoula chamado comercialmente de Lipiodol ${ }^{\circledR}$ (laboratório Guerbet) por ser do tipo oleoso e por permanecer durante mais tempo no sistema lacrimonasal para a realização dos exames. 0 exame dacriocistográfico representa o estudo da imagem da via lacrimal por injeção de contraste no interior da mesma, sendo possível empregar contraste hidrossolúvel ou lipossolúvel (Weil 1988). 0 lipossolúvel é de eliminação mais lenta, já que não se mistura à lágrima, e delimita muito melhor o contorno das estruturas, sendo melhor para avaliação das vias lacrimais. Porém, o tempo de permanência nos cães não foi grande, sendo necessária administração contínua manual durante o exame radiográfico e injeção contínua com uso de bomba de infusão peristáltica durante o exame de tomografia computadorizada.

Neste estudo, os exames radiográficos foram feitos com os animais em posição látero-lateral, e os tomográficos foram executados com os cães em decúbito dorsoventral, ambos com os pacientes sob anestesia geral. Não se obteve êxito na tentativa de posicionar os animais com a boca aberta em decúbito ventro-dorsal em ambos os exames supracitados, nem com pálato duro paralelo à mesa para obtenção de imagem transversa da cavidade nasal com os pacientes em decúbito dorsal por elevação do nariz com o pálato duro, preferencialmente, perpendicular à mesa de scanner, pois o contraste espalhava-se na região de seios nasais e dificultava a visibilização das imagens. Slatter (1990a) relatou que, em cães, o estudo radiográfico contrastado é realizado com o paciente sob anestesia geral e em decúbito lateral. Pode ser realizada também, exposição radiográfica dorso-ventral. Segundo Gelatt (2003), existe a possibilidade de realizar-se radiografias nasais com a boca aberta, lateral e ventro-dorsal.

No estudo, optou-se por realizar a dacriocistografia, um lado de cada vez, devido ao rápido escoamento do contraste pelo sistema lacrimal e ao posicionamento do animal ser látero-lateral, dificultando a canulação e injeção do contraste no ponto lacrimal contralateral em contato direto com a mesa. Segundo Gelatt (2003), geralmente $1 \mathrm{ml}$ de contraste iodado viscoso é simultaneamente injetado em ambos os lados e radiografias dorso-ventrais são feitas e, às vezes, lateral. Cinco minutos após a injeção, é realizada nova radiografia para avaliar o efeito gravitacional sobre a drenagem lacrimal.

No presente estudo, houve a tentativa de evitar o contato do contraste com os pelos, o que atrapalharia a visibilização dos exames por impregnação de outras estruturas, utilizando algodão e gaze, sem sucesso. Esta foi a técnica sugerida por Slatter (1990a) que colocou algodão e gaze também dentro das narinas externas, próximo ao óstio nasal do sistema lacrimonasal, para prevenir ou limitar o extravasamento de meio de contraste nos pelos faciais e absorver o excesso drenado. Assim, optou-se pela aplicação de esparadrapo logo abaixo das pálpebras inferiores e em focinho, delimitando inferiormente os pontos lacrimais inferiores, recoberto por contact, o que facilitou a limpeza do mesmo, evitando o obscurecimento da imagem radiográfica e tomográfica.
No presente estudo, a dilatação foi a alteração mais observada pelos exames de dacriocistografia e tomografia computadorizada. Esta alteração esteve presente no GE nas quatro regiões descritas, sendo que o maior número de casos ocorreram na região 4 (76,3\% no R-X e $84,2 \%$ na TC) indicando que a dilatação se dá principalmente na porção distal da via lacrimal excretora. Ressalta-se, entretanto, que somente nessa mesma região, os animais do GC também a apresentaram, embora em percentual bastante reduzido em relação ao anterior (RX: 12,5\%; TC: 3,1\%).

Outra aspecto observado foi a comunicação do DLN com os seios nasais na região 3, tanto no GE (RX: 55,3\%; TC: 73,7\%) quanto no GC (R-X: 46,9\%; TC: 68,7\%), demonstrando que a comunicação pode estar presente em cães com ou sem epífora, podendo indicar que tal alteração seja própria de alguns cães, não necessariamente levando ao desenvolvimento de alguma afecção, como por exemplo, a epífora.

As demais alterações tais como: obstrução, massa, tortuosidade do ducto do sistema de drenagem lacrimal dos cães estudados foram observadas com bem menos frequência e estenose em ambos os grupos.

\section{CONCLUSÕES}

Animais com sinal clínico de epífora apresentam alterações radiográficas e tomográficas no DLN, principalmente dilatação em regiões mais distais;

Dilatações também podem ser observadas em alguns cães sem sinal clínico da afecção;

Comunicação do ducto lacrimonasal com o seio nasal não indica alteração causadora de epífora, pois está presente em animais com e sem afecção;

Os dados do presente estudo confirmam que o exame DCG por si pode dar importantes informações, de forma semelhante à TC, devendo-se recorrer à ela apenas quando houver dúvidas sobre lesões ósseas, fraturas e corpos estranhos não detectados pelo primeiro.

Agradecimentos.- Ao Laboratório Guerbet pela disponibilização do meio de contraste Lipiodol ${ }^{\circledR}$, permitindo a realização deste trabalho, ao anestesista Eulálio L.M. Pimenta que esteve presente durante todo o trabalho fazendo com que nossos cães fossem anestesiados com toda segurança, ao Departamento de Cirurgia e Anestesiologia e ao Departamento de Radiologia Veterinária que sem eles seria impossível a realização do projeto, a Unesp Campus Botucatu por me acolher e ao Conselho Nacional de Desenvolvimento Científico e Tecnológico (CNPq) que forneceu uma bolsa de estudos necessária a minha manutenção durante o mestrado.

\section{REFERÊNCIAS}

Araf D. 2004. Propedêutica de vias lacrimais, p.217-224. In: Matayoshi S., Forno E. \& Moura E.M. (Eds), Manual de Cirurgia Plástica Ocular. Vol.7. Roca, São Paulo. (Atualidades em Oftalmologia)

Barnett K.C., Crispin S.M., Lavach J.D. \& Mattews A.G. 1995. Lacrimal system, p.75-83. In: Ibid. (Eds), Color Atlas and Text of Equine Ophthalmology. Mosby-Wlfe, London.

Böhme G. 1992. Tränenapparat, Apparatus lacrimalis, p.435-437. In: Nickel R., Schummer A. \& Seiferle E. (Eds), Lehrbuch der Anatomie der Haustiere. Parey, Berlin.

Boruchoff S.A. \& Boruchoff S.E. 1992. Infections of the lacrimal system. Infect. Dis. Clin. North Am. 6(4):925-932.

Carneiro Filho L. 1997. Manual de Oftalmologia Veterinária: um guia prático para clínicos veterinários. Roca, São Paulo. 120p.

Caye E.A., Maciel A.C., Teixeira T. \& Ilha D.O. 1986. Dacriocistografia no estudo da epífora em 63 pacientes. Revta Imagem 8(3):107-112. 
Costa M.N., Schellini S.A. \& Moura E.M. 1997. Conjuntivodacriocistorrinostomia, p.263-270. In: Soares E.J.C., Moura E.M. \& Gonçalves J.O.R. (Eds), Cirurgia Plástica Ocular. Vol.8. Roca, São Paulo.

Covitz D., Hunziker J. \& Kich S.A. 1977. Conjunctivorhinostomy: a surgical method for the control of epiphora in the dog and cat. J. Am. Vet. Med. Assoc. 171:251-255.

Czyz C.N., Bacon T.S., Stacey A.W., Cahill E.N., Costin B.R., Karanfilov B.I. \& Cahill K.V. 2015. Nasolacrimal system aeration on computed tomographic imaging: effects of patient positioning and scan orientation. Clin. Ophthalmol., Auckland, 9:469.

Diesem C. 1981. Generalidades sobre órgãos sensoriais e integumento comum: o órgão da visão, p.207-222. In: Getty R. (Ed.), Sisson/Grossman Anatomia dos Animais Domésticos. Vol.1. 5 ${ }^{\underline{a}}$ ed. Interamericana, Rio de Janeiro.

Gelatt K.N., Cure T.H. \& Guffy M.M. 1972. Dacryocystorhinography in the dog and cat. J. Small Anim. Pract. 13:381-397.

Gelatt K.N. 2003. Doenças e cirurgia dos sistema lacrimal e nasolacrimal do cão, p.73-94. In: Ibid. (Ed.), Manual de Oftalmologia Veterinária. Manole, São Paulo.

Grahn B.H. \& Mazson R.A. 1995. Epiphora associated with dacryops in a dog. J. Am. Anim. Hosp. Assoc. 31(1):15-19.

Groell R., Schaffler G.J., Uggowitzer M., Szolar D.H. \& Muellner K. 1997. CT-anatomy of the nasolacrimal sac and duct. Surgical and Radiologic Anatomy 19(3):189-191.

Habin D. 1993. The nasolacrimal system, p.91-102. In: Petersen-Jones S.M. \& Crispin S.M. (Eds), Manual of Small Animal Ophthalmology. Brit. Small Anim. Vet. Assoc., Shurdington.

Kanski J.J. 2004a. Distúrbio do sistema de drenagem lacrimal, p.43-54. In: Ibid. (Ed.), Oftalmologia Clínica: uma abordagem sistemática. $4^{\underline{a}}$ ed. Revinter, Rio de Janeiro.

Kanski J.J. 2004b. Sistema de drenagem lacrimal, p.43-55. In: Ibid. (Ed.), Oftalmologia Clínica: uma abordagem sistemática. 5a ed. Elsevier, Rio de Janeiro.

Kleiner J.A. 2003. Tratamento cirúrgico da epífora crônica em animais de companhia. Dissertação de Mestrado, Setor de Ciências Agrárias, Escola de Agronomia e Veterinária, Universidade Federal do Paraná, Curitiba. 57p.

Kleiner J.A., Wouk A.F.P.F., Costa P.V. \& Fidelcino A. 2004. A dacriocistorrinografia em cães e gatos. Medvep Revta Cient. Med. Vet., Peq. Anim. Estim. 2(7):185-191.

Lee H., Ha S., Le Y., Park M. \& Bael S. 2011. Anatomical and morphometric study of the bony nasolacrimal canal using computed tomography. Ophthalmologica 227(3):153-159.

Lefebvre D.R. \& Freitag S.K. 2012. Update on imaging of the lacrimal drainage system. Seminars in Ophthalmology, Informa Healthcare, New York, p.175-186.

Lloyd G.A. \& Welham R.A. 1974. Substraction macrodacryocystography. Brit. J. Radiol. 47(559):379-382.

Long R.D. 1975. The relief of epiphora by conjunctivorhinostomy. J. Small Anim. Pract. 16:381-386.

Michel G. 1955. Beitrag zur Anatomie der Tränenorgane von Hund und Katze. Dtsch. Tierärztl. Wochenschr. 62:347-349.

Mills D.M. \& Meyer D.R. 2006. Acquired nasolacrimal duct obstruction. Clin. North Am., Otolaryngol. 39:979-999.
Nykamp S.G., Scrivani P.V. \& Pease A.P. 2004. Computed tomography dacryocystography evaluation of the nasolacrimal apparatus. Vet. Radiol. Ultrasound 45(1):23-31.

Pereira L., Dammann F., Duda S.H., Reinbold W.D. \& Claussen C.D. 1997. Value of dacryocystography in localization diagnosis of lacrimal duct. Stenosis 166(6):498-501.

Rached P.A., Canola J.C., Schlüter C., Laus J.L., Oechtering G., Almeida D.E. \& Ludewig E. 2011. Computed tomographic-dacryocystography (CT-DCG) of the normal canine nasolacrimal drainage system with three-dimensional reconstruction. Vet. Ophthalmol. 14(3):174-79.

Rached P.A., Ludewig E., Oechtering G. \& Noller C. 2007. CT- and MRDacryocystography of the normal canine nasolacrimal drainage system: preliminary results in 15 dogs. Annual Meeting, Porto Carras. (Abstracts)

Ramey N.A., Hoang J.K. \& RIichard M.J. 2013. Multidetector CT of nasolacrimal canal morphology: normal variation by age, gender, and race. Ophthalmic Plastic and Reconstructive Surgery 29(6):475-480.

Roberts S.R., Vierheller R.C. \& Lennox W.J. 1974. Eyes, p.193-262. In: Archibald J. (Ed.), Canine Surgery. 2nd ed. American Veterinary Publications, Santa Barbara.

Severin G.A. 1991. Aparato lacrimal, p.85-97. In: Ibid. (Ed.), Manual de Oftalmologia Veterinária. Hemisfério Sur, Buenos Aires.

Schellini S.A., Hercules L.A., Padovani C.R., Nascimento S.M., Lopes P.S. \& Schellini R.C. 2005. Dacriocistografia na propedêutica via lacrimal excretora de adultos. Arq. Bras. Oftalmol. 68(1):89-92.

Shankar J., Gupta S.R. \& Walsh P. 1999. Dilation and stenting for naso-lacrimal duct obstruction: a pilot projetct. Acta Ophthalmologica Scandinavica 77(5):555-558.

Silva J.A.F. 2004. Alterações do sistema lacrimal, p.51-56. In: Schor P., Chamon W. \& Belfort Jr R. (Eds), Guias de Medicina Ambulatorial e Hospitalar. Unifesp/Escola Paulista de Medicina: guia de oftalmologia. Manole, São Paulo.

Slatter D. 1990a. Basic diagnostic techniques, p.84-123. In: Ibid. (Ed.), Fundamentals of Veterinary Ophthalmology. 2nd ed. W.B. Saunders, Philadelphia.

Slatter D. 1990b. Lacrimal system, p.237-256. In: Ibid. (Ed.), Fundamentals of Veterinary Ophthalmology. 2nd ed. W.B. Saunders, Philadelphia.

Stades F.C., Boevé M.H., Neuman W. \& Wyman M. 1999. Fundamentos da Oftalmologia Veterinária. Manole, São Paulo. 204p.

Takano B.A. \& Mendonça Junior A.A. 1996. Dacriocistografia: aspectos radiológicos nas alterações das vias lacrimais: análise de 24 casos. Radiol. Bras. 29(1):23-29.

Wearne M.J., Pitts J., Frank J. \& Rose G.E. 1999. Comparison of dacryocystography and lacrimal scintilography in the diagnosis of function nasolacrimal duct obstruction. Brit. J. Ophthalmol. 83(9):1032-1035.

Weil B. 1988. Vias lacrimais. Anais Oftalmol. 8(1):26-32.

Winston S. \& Prasse K.W. 2003. Doenças oftálmicas, p.446-475. In: Fenner W.R. (Ed.), Consulta Rápida em Clínica Veterinária. 3ª ed. Guanabara Koogan, Rio de Janeiro.

Yu G., Zhang C.Y., Cui Y.H., Fan Y.W., Cao W.H., Lin Q., Qi Y., Cui J., Hu M., Liu W. \& Wu Q. 2013. Application value of computed tomography dacryocystography in children lacrimal diseases [Zhonghua yan ke za zhi]. Chinese J. Ophthalmol. 49(8):706-710.

Zaldivar R.A., Buerger D.E., Buerger D.G. \& Wogg J.J. 2006. Office evaluation of lacrimal and orbital disease. Clin. North Am., Otolaryngol. 39:911-922. 